\title{
BMJ Open Impact of restricted visitation policies in hospitals on patients, family members and healthcare providers during the COVID-19 pandemic: a scoping review protocol
}

To cite: Moss SJ, Stelfox HT, Krewulak KD, et al. Impact of restricted visitation policies in hospitals on patients, family members and healthcare providers during the COVID-19 pandemic: a scoping review protocol. BMJ Open 2021;11:e048227. doi:10.1136/ bmjopen-2020-048227

- Prepublication history and additional supplemental material for this paper are available online. To view these files, please visit the journal online (http://dx.doi.org/10.1136/ bmjopen-2020-048227).

JPL and KMF are joint senior authors.

Received 22 December 2020 Accepted 10 September 2021

Check for updates

(c) Author(s) (or their employer(s)) 2021. Re-use permitted under CC BY-NC. No commercial re-use. See rights and permissions. Published by BMJ.

For numbered affiliations see end of article.

Correspondence to Dr Kirsten M Fiest; kmfiest@ucalgary.ca

\section{ABSTRACT}

Introduction Flexible visitation policies in hospitals are an important component of care that contributes to reduced stress and increased satisfaction among patients and their family members. Early evidence suggests restricted visitation policies enacted in hospitals during the COVID-19 pandemic are having unintended consequences on patients, family members and healthcare providers. There is a need for a comprehensive summary of the impacts of restricted visitation policies on key stakeholders and approaches to mitigate that impact.

Methods and analysis We will conduct a scoping review as per the Arksey-0'Malley 5-stage scoping review method and the Scoping Review Methods Manual by the Joanna Briggs Institute. We will search relevant electronic databases (eg, CINAHL, MEDLINE, PsycINF0), grey literature and preprint repositories. We will include all study designs including qualitative and quantitative methodologies (excluding protocols) as well as reports, opinions and editorials, to identify the broad impact of restricted hospital visitation policies due to the COVID-19 pandemic on patients, family members or healthcare providers of hospitalised patients, and approaches taken or proposed to mitigate this impact. Two reviewers will calibrate the screening criteria and data abstraction form and will independently screen studies and abstract the data. Narrative synthesis with thematic analysis will be performed.

Ethics and dissemination Ethical approval is not applicable as this review will be conducted on published literature only. This scoping review will identify, describe and categorise impacts of restricted hospital visitation policies due to the COVID-19 pandemic on patients, family members and healthcare providers of hospitalised patients, and approaches that have been taken to mitigate impact. We will provide a comprehensive synthesis by

\section{Strengths and limitations of this study}

- We will conduct a comprehensive literature search of multiple electronic databases and sources for difficult to locate unpublished records.

- Our scoping review will conform to the Arksey0'Malley 5-stage scoping review method and the manual by the Joanna Briggs Institute.

- We will include all study designs including qualitative and quantitative methodologies as well as reports, opinions and editorials, to identify the broad impact of restricted hospital visitation during the first and second waves of the pandemic.

- The literature searches might be more extensive than anticipated, given the rapid and continuous nature of COVID-19 research.

- We anticipate challenges related to categorising restricted visitation policies accurately and outlining approaches that have been taken to mitigate impact.

developing a framework of restricted visitation policies and associated impacts. Our results will inform the development of consensus statements on restricted visitation policies to be implemented in future pandemics. PROSPERO registration number CRD42020221662.

\section{BACKGROUND}

Guidelines recommend flexible visitation policies in hospitals as an important component of quality care $^{12}$ that contributes to reduced stress ${ }^{34}$ and increased satisfaction ${ }^{3}$ among patients and their family members. The COVID-19 pandemic has resulted in restrictions to visitation for hospitalised 
patients in order to limit the spread of COVID-19, organise care, and to reduce the use of personal protective equipment. ${ }^{56}$ Given the important role of family visitation and engagement in patient-centred and family-centred care, ${ }^{7}$ early evidence suggests that restricted visitation policies enacted in hospitals during the COVID-19 pandemic will have unintended consequences on patients, family members, and healthcare providers. ${ }^{8}$

Several psychological interventions for people affected by COVID-19 have been taken since the start of the pandemic, including assessment of risk factors in the development of mental health disorders, prevention of injury to self and bereavement therapy for family members grieving lost loved ones. ${ }^{9}{ }^{10}$ It has been suggested that the mental health needs of patients with suspected or confirmed COVID-19, their family members and healthcare providers, have been poorly handled. ${ }^{11}$ To date, no comprehensive summary has assessed the impacts of restricted visitation policies and the approaches that have been taken to mitigate this impact. This evidence gap may constitute a barrier to understanding the best way to effectively manage humane interaction and communication when restrictions on visitation are required.

We report a protocol for a scoping review designed to identify, describe and categorise impacts of restricted hospital visitation policies due to the COVID-19 pandemic on patients, family members or healthcare providers of hospitalised patients, and approaches taken to mitigate impact. The results of our scoping review will directly inform future strategies to mitigate the impacts of COVID-19 and will be transferable to future pandemic care responses.

\section{Review questions}

This scoping review will be conducted as per the ArkseyO'Malley 5-stage scoping review method ${ }^{12}$ and the Scoping Review Methods Manual by the Joanna Briggs Institute. ${ }^{13}$ The Preferred Reporting Items for Systematic Review and Meta-Analysis Protocols guideline was used to develop the protocol ${ }^{14}$ and was registered on PROSPERO prior to data extraction. We will adhere to the Preferred Reporting Items for Systematic Reviews and Meta-Analyses (PRISMA)-ScR Extension for Scoping Reviews ${ }^{15}$ to report findings.

This scoping review has two objectives:

1. What are the impacts of restricted hospital visitation policies due to the COVID-19 pandemic on patients, family members or healthcare providers of hospitalised patients?

2. What approaches have been taken to mitigate the impact on patients, family members or healthcare providers?

The components of population, exposure, comparator, outcome, study design and timeframe are as follows:

- Population: patients, family members or healthcare providers of adult patients hospitalised at acute care facilities during the COVID-19 pandemic.
- Exposure: restricted visitation for hospitalised patients because of the COVID-19 pandemic.

- Comparator: any comparator.

- Outcomes: perspectives (ie, views or prospects), experiences (ie, encounters) or quantitative impacts (eg, any diagnosed psychopathologies or symptoms of psychopathologies, diagnosed neurocognitive disorders or symptoms of neurocognitive disorders, health-related quality of life, self-efficacy (ability to function and maintain relationships), general wellbeing (coping, sense of meaning, purpose, optimism and hopefulness)) among patients, family members or healthcare providers, as well as approaches or strategies taken to mitigate impact (eg, education sessions, informal or formal support groups, (TeleHealth or Mobile Health) communication platforms).

- Study design: any observational or interventional primary research study, including focus groups and qualitative inquires, as well as reviews, commentaries, editorials, opinions, case studies and case reports or reports from expert advisory groups.

- Timeframe: publications from 1 December 2019 to 1 March 2021.

\section{METHODS AND DESIGN}

\section{Study designs eligible}

We will include any published or preprint article that reports novel findings and exceeds 500 words, including but not limited to primary observational studies (eg, cross-sectional (surveys), cohort, case-control studies, including focus groups and qualitative inquiries), interventional studies (eg, randomised controlled trials), as well as reviews, commentaries, editorials, opinions, case studies and case reports, and reports from expert advisory groups or panels (eg, Re-Integration of Family Caregivers as Essential Partners in Care in a Time of COVID-19; Canadian Foundation for Healthcare Improvement) (table 1).

\section{Participant eligibility}

Eligible study populations will include patients, family members (ie, relatives, close friends) or healthcare providers (eg, nurses, physicians, respiratory therapists) of adult patients $(>17$ years of age, or as defined in the individual study) who were hospitalised at acute care facilities (ie, tertiary academic or community hospitals, or specialised care centres part of a larger hospital) during the COVID-19 pandemic. We will include both patients with COVID-19-positive and COVID-19-negative, and outcomes of these groups will be stratified if reported. We will exclude family members and healthcare providers of hospitalised children as we expect the restricted visitation policies and exceptions to these policies to vary according to the patient-family member/provider relationship. This research builds on our environmental scan that describes the extent, variation and fluctuation of Canadian hospital visitation policies before and during the COVID-19 pandemic (in preparation). 
Table 1 Inclusion and exclusion criteria for a scoping review investigating the impact of restricted visitation policies in hospitals enacted because of the COVID-19 pandemic on patients, family members and healthcare providers of hospitalised patients, and the approaches that have been taken to mitigate impact

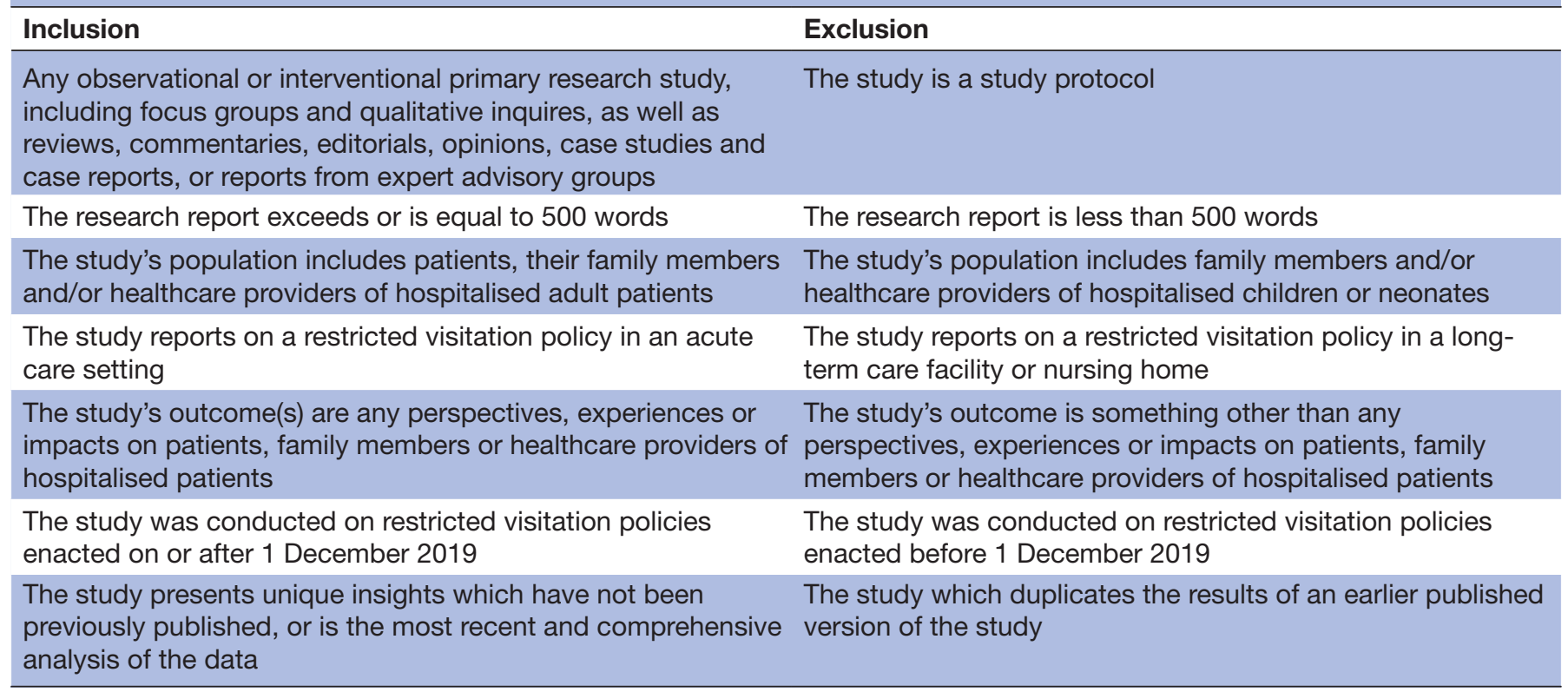

\section{Exposures eligible}

The exposure is restricted visitation in acute care facilities (ie, tertiary academic and community hospitals (including specialised care centres)) during the COVID-19 pandemic. We will exclude studies that report on a restricted visitation policy in a specialised care centre that is separate from a larger hospital, or in a long-term care facility (eg, senior residences). We will compare exposures based on degree of restriction (ie, highly restrictive (no family members per patient) compared with less restrictive (one or more family members per patient)), as well as based on COVID-19 status of the patient (ie, COVID-19-positive or COVID-19-negative).

\section{Outcome measures eligible}

All eligible studies will present perspectives (ie, views or prospects), experiences (ie, encounters) or impacts (defined below) of restricted visitation policies on the patients, family members or healthcare providers of hospitalised adult patients during the COVID-19 pandemic. Eligible studies do not need to characterise approaches or strategies to mitigate impact of restricted visitation policies (eg, education sessions, informal or formal support groups, (TeleHealth or Mobile Health) communication platforms), though these studies will also be included.

We will include studies that report on any impact of restricted visitation policies on the patients, family members or healthcare providers of hospitalised adult patients (both COVID-19-positive and COVID-19negative) during the COVID-19 pandemic, including but not limited to any diagnosed psychopathologies or symptoms of psychopathologies, any diagnosed neurocognitive disorders or symptoms of neurocognitive disorders, quality of life or health-related quality of life, self-efficacy (ie, ability to function and maintain relationships), and general well-being (ie, coping, sense of meaning, purpose, optimism and hopefulness) measurements. Given the expectation that restricted visitation policies will have psychological and cognitive mediators, studies that report on any outcome related to mental health or well-being will be eligible for inclusion. As we expect outcome measures to vary and use different measurement tools, we will not prespecify principal summary measures. The overall findings in relation to perspectives, experiences and impacts, will be summarised individually and collectively. We will not combine included studies in meta-analyses given our desire to understand and interpret the variation in restricted visitation policies enacted between different hospital settings. Rather, we will summarise studies using narrative synthesis.

\section{Timeframe eligible}

Eligible studies will be publications from 1 December 2019 to 1 March 2021. No upper limit will be applied, and searches will be updated prior to manuscript submission.

\section{Search methods for identification of studies}

Articles for this review will be identified in CINAHL, Cochrane Databases of Reviews and Clinical Trials, EMBASE, Healthstar, MEDLINE and PsycINFO. The search strategy for MEDLINE was developed by a librarian working with the investigators and approved by a second librarian through completion of a Peer Review of an Electronic Search Strategy (PRESS) checklist. ${ }^{16}$ We will not apply limiters or restrictions (eg, date, publication type) to any of the searches. A range of broad search terms will be used to maximise the yield of the search for studies. Search terms were selected based on components 
of restricted visitation policies in acute care facilities during the COVID-19 pandemic, and family member or healthcare provider perspectives, experiences and impacts. The full search strategy for the MEDLINE database can be found in online supplemental appendix 1 . To include relevant information from unpublished sources, we will perform manual searches in web-based resources, including: Google, Google Scholar, journals which published key articles (eg, Lancet, New England Journal of Medicine, Journal of the American Medical Association), and through searching specific websites (ie, Centers for Disease Control and Prevention, https://www.cdc.gov; National Institute for Communicable Disease, https:// www.nicd.ac.za; National Institute for Health and Clinical Excellence, https://www.nice.org.uk; National Health Commission of the People's Republic of China, http:// www.nhc.gov; and National Administration of Traditional Chinese Medicine, http://www.satcm.gov.cn; WHO, https://www.who.int). We will also perform manual searches in ProQuest (for theses and dissertations) and medRxiv (https://www.medrxiv.org; preprint server for health sciences). The terms "COVID-19" and "Hospital Policy" will be searched separately in all manual databases and the first five pages (based on relevancy) will be screened for potentially relevant articles.

\section{Selection of eligible studies}

At the title and abstract screening stage, a subset of the team (SM, KM, LH) will achieve $100 \%$ agreement on a calibration exercise of 50 random citations prior to commencing selection of eligible studies. After reliability in reference screening is ensured, two reviewers $(\mathrm{SM}$; KF, $\mathrm{KM}$ or LH) will independently screen titles and abstracts for potential inclusion using screening questions developed for this review (table 2). Any study selected by any reviewer at this stage will progress to the next stage. After initial screening, two authors (SM; KM or LH or MA) will examine full-texts independently and in duplicate for eligibility and for development of the final data abstraction table. A separate calibration exercise will be then performed for screening reference lists of selected articles. (eg, literature reviews or summary reports on this topic). Two independent authors (SM; KM or LH or MA) will screen reference lists of selected articles to identify relevant articles for potential study selection; original articles will be sourced, and the full-text assessed for eligibility. Disagreements in study selection at the full-text stage will be resolved by a third reviewer (KK).

\section{Data abstraction from included studies}

Data abstraction will be conducted by the same authors who performed the screening and full-text review (SM; $\mathrm{KM}$ or LH or MA), and will include study identifiers and study design, participants, exposure and outcome information, as well as information on approaches or strategies taken to mitigate impact and author conclusions and recommendations (table 3). Missing information will be noted. Data abstracted regarding changes to hospital visitation restriction will be categorised according to the restricted visitation policy tree (figure 1). Approaches or strategies to mitigate impact will be summarised qualitatively in thematic analysis, for which the process is described below.

\section{Process for data abstraction}

A data abstraction form will be created in Microsoft Excel. The abstraction form will be piloted and tested by the data abstractors (SM, KM, LH and MA) on a subset of studies (ie, $5 \%$ of studies if $n>50,10 \%$ of studies if $n \leq 50$ ) to ensure clarity. Following pilot testing, the form will be adapted as recommended by the abstractors to improve usability and completeness. The first author (SM) and one additional abstractor (KM or LH or MA) will complete data extraction. Data abstraction will be completed independently; the first author will complete all data abstraction for all included studies, and the additional abstractor will check and compare the abstracted data for accuracy. Disagreements will be resolved by a third reviewer (KK). If data presented in a study is unclear, missing or presented in a format that is unusable or cannot be abstracted, we will attempt to contact corresponding authors for clarification. We will contact authors via email, and a follow-up email will be sent 2 weeks later. We will allocate a 4 -week period to receive responses from authors beginning from the time of the initial contact attempt. Thereafter, we will document missing data in the review. We will not appraise methodological quality because the aim of a scoping review is to identify gaps in the evidence base and target topics for future reviews.

\section{Strategies for data synthesis}

Data synthesis will be performed according to a twostage process to address the two study objectives. Table 4 shows the two-stage synthesis plan, questions to guide synthesis and anticipated outputs for both of the research questions. Data synthesis for each research question will involve both quantitative analysis (eg, frequencies, proportions, missing data for descriptive statistics) and qualitative analysis (ie, thematic synthesis for reviews on health research ${ }^{17}$ ). Thematic synthesis will include three stages: (1) inductive coding of text line-by-line; (2) development of descriptive themes and (3) generation of analytical themes. We will develop discrete themes that validly and accurately represent the themes reported by the primary studies; our analytical themes will represent a stage of interpretation whereby we will go beyond the primary studies to generate new interpretive constructs, explanations or hypothesis. We expect that this multilayer method to data synthesis will allow us to maintain a clear and transparent link between our conclusions and the published results or insights of the articles, and to reveal potential topics for other systematic reviews or future novel research.

\section{Presentation of the results}

The final search strategy for each database and all ancillary searches conducted will be provided as an online 
Table 2 Screening questions to identify literature for including in a scoping review investigating the impact of restricted visitation policies in hospitals enacted because of the COVID-19 pandemic on patients, family members and healthcare providers of hospitalised patients, and the approaches that have been taken to mitigate impact

\begin{tabular}{|c|c|}
\hline \multirow{2}{*}{$\begin{array}{l}\text { Screening domain and } \\
\text { question }\end{array}$} & Characteristics for assessme \\
\hline & Yes-include \\
\hline \multicolumn{2}{|c|}{ Title and abstract screening question to be used to identify lite } \\
\hline $\begin{array}{l}\text { Study design: does the } \\
\text { title/abstract describe an } \\
\text { observational or interventional } \\
\text { primary research study, } \\
\text { including focus groups and } \\
\text { qualitative inquires, as well } \\
\text { as reviews, commentaries, } \\
\text { editorials, opinions, case } \\
\text { studies and case reports, or } \\
\text { reports from expert advisory }\end{array}$ & $\begin{array}{l}\text { Yes: the study employs an } \\
\text { observational or interventional } \\
\text { primary research design, } \\
\text { including focus groups and } \\
\text { qualitative inquires, as well } \\
\text { as reviews, commentaries, } \\
\text { editorials, opinions, case } \\
\text { studies and case reports, or } \\
\text { reports from expert advisory } \\
\text { groups. }\end{array}$ \\
\hline
\end{tabular}
groups?

\begin{tabular}{|c|c|}
\hline $\begin{array}{l}\text { Publication type: does the } \\
\text { title and abstract come from a } \\
\text { published study, government } \\
\text { report, non-government } \\
\text { report or postsecondary } \\
\text { institutional thesis (exceeding } \\
500 \text { words in length)? }\end{array}$ & $\begin{array}{l}\text { Yes: the study is a published } \\
\text { study, government report, } \\
\text { non-government report or } \\
\text { postsecondary institutional } \\
\text { thesis (exceeding } 500 \text { words } \\
\text { in length). }\end{array}$ \\
\hline
\end{tabular}

Population: does the population of interest include patients, family members and/ or healthcare providers of hospitalised adult patients?

$\begin{array}{ll}\begin{array}{l}\text { Exposure: is the study's } \\ \text { exposure of interest an } \\ \text { enacted policy on restricted } \\ \text { visitation for hospitalised } \\ \text { patients? }\end{array} & \begin{array}{l}\text { Yes: the exposure of interest } \\ \text { is an enacted policy on } \\ \text { restricted visitation for } \\ \text { hospitalised patients. }\end{array} \\ \begin{array}{ll}\text { Outcome: does the title } \\ \text { and/or abstract describe } \\ \text { research on the perspectives, }\end{array} & \begin{array}{l}\text { Yes: one or more of the } \\ \text { oxperiences or impacts }\end{array} \\ \begin{array}{l}\text { rexperiences in the study are } \\ \text { of restricted visitation in }\end{array} & \begin{array}{l}\text { experts } \\ \text { of restricted visitation in }\end{array} \\ \text { hospitals on patients, family } & \text { hospitals on patients, family } \\ \text { members or healthcare } & \text { members or healthcare } \\ \text { providers? } & \text { providers. }\end{array}$

\section{Screening domain and question}

Yes: the study population describes patients, family members and/or healthcare providers of hospitalised adult patients.

Yes: the exposure of interest restricted visitation for Yes: one or more of the outcomes in the study are pectives, periences or impacts hospitals on patients, family providers.
No-exclude

\section{Unclear-include}

No: the study is not a published study, government report, non-government report or postsecondary institutional thesis or is fewer than 500 words in length.
Unclear: it is unclear if the study is a published study, or government report, nongovernmental report or postsecondary institutional thesis (exceeding 500 words in length) from the title and abstract.

No: the population of interest Unclear: it is unclear if the is not entirely patients, family population of interest is members and/or healthcare patients, family members and/ providers of hospitalised adult or healthcare providers of patients.

No: the exposure of interest is not an enacted policy on restricted visitation for hospitalised patients.

No: there is no outcome in the study related to any of perspectives, experiences or impacts of restricted visitation in hospitals on patients, family members or healthcare providers. hospitalised adult patients.

Unclear: it is unclear if the exposure of interest is an enacted policy on restricted visitation for hospitalised patients.

Unclear: it is unclear from the title and abstract if the outcome of interest is related to any perspectives, experiences or impacts of restricted visitation in hospitals on patients, family members or healthcare providers.

Characteristics for assessment

$$
\text { Yes-include No-exclude }
$$

Full article screen questions to be used to identify literature for inclusion in the systematic review

${ }^{*}$ Full article screening questions include all screening domains and questions from title and abstract screening, and two

\begin{tabular}{|c|c|c|}
\hline $\begin{array}{l}\text { Time frame: was the restricted visitation policy enacted on or } \\
\text { after } 1 \text { December } 2019 ?\end{array}$ & $\begin{array}{l}\text { Yes: the restricted visitation } \\
\text { policy was enacted on or after } \\
1 \text { December } 2019 \text {. }\end{array}$ & $\begin{array}{l}\text { No: the restricted visitation } \\
\text { policy was enacted before } 1 \\
\text { December } 2019 \text {. }\end{array}$ \\
\hline $\begin{array}{l}\text { Setting: was the setting where the restricted visitation policy } \\
\text { was enacted an acute care facility? }\end{array}$ & $\begin{array}{l}\text { Yes: the setting where the } \\
\text { restricted visitation policy } \\
\text { was enacted is an acute care } \\
\text { facility. }\end{array}$ & $\begin{array}{l}\text { No: the setting where the } \\
\text { restricted visitation policy was } \\
\text { enacted is a long-term care } \\
\text { facility or a nursing home. }\end{array}$ \\
\hline
\end{tabular}
additional domains 
Table 3 Data to be abstracted from eligible studies included in the review

\section{Data domain Data categories}

Study identifiers: from a published study, government Authors' names; study title; publication type; publication date; journal, report, non-government report or postsecondary volume, issue and page numbers of publication; place of publication institutional thesis (exceeding 500 words in length) (ie, first author's institutional email address) and digital object identifier

Study design: primary research, review, commentary, Study type or design; time frame of study; location of study (ie, country) editorial, opinion, case study, case report or report and region of study (localised when reported) from an expert advisory groups

Participants: family members and/or healthcare providers of hospitalised adult patients

Definition and size (ie, N) of the source population(s) at risk; relevant demographic information (eg, age, gender, relationship to patient); patient admitting diagnosis; patient hospital length of stay; patient intensive care unit length of stay (if applicable); patient mortality (if applicable)

Exposure: enacted policy on restricted visitation for hospitalised patients as a result of COVID-19 at an acute care facility

Outcome: perspectives, experiences, impacts
Type and catchment area of hospital; date of enactment; visitation policies prior to the COVID-19 pandemic; extent of changes to visitation policies*

Patient COVID-19 status; assessment tool(s); timepoint of assessment(s); perspectives (ie, mental views or prospects); experiences (ie, encounters); impacts (eg, any diagnosed psychopathologies or symptoms of psychopathologies, neurocognitive disorders or symptoms of neurocognitive disorders; healthrelated quality of life, self-efficacy (ability to function and maintain relationships), general well-being(coping, sense of meaning, purpose, optimism and hopefulness)); approaches or strategies taken to mitigate impact (eg, education sessions, informal or formal support groups, (TeleHealth or Mobile Health) communication platforms); statistical significance; authors' conclusions and recommendations

${ }^{*}$ Data will be categorised according to the restricted visitation policy tree.

supplemental appendix in the final publication. A flow chart, following the PRISMA-ScR Extension for Scoping Reviews, ${ }^{15}$ will illustrate where citations were eliminated during screening and ancillary searches, including rationale for exclusion in full-text screening. All included studies will be presented in a narrative synthesis. A table of characteristics of included studies will be included in the published journal article and will describe study identifiers and period of study, sample and setting, exposure, direction, and magnitude of the outcome(s), approaches to mitigate impact, and authors' conclusions and recommendations. Descriptive statistics (ie, frequencies, proportions, missing data) will be provided as extensions of this table when appropriate or in the narrative synthesis. Thematic synthesis (ie, our developed analytical themes) will be presented as a separate table and summarised narratively in the results.

\section{Patient and public involvement}

Patient and family partnership in health research and clinical care is essential to achieve patient and family-centred care. ${ }^{18}$ The proposed project will abide by the guiding core principles of inclusiveness, mutual respect, support and co-building. ${ }^{19}$ Patient (MA, NF) and family member (BS) involvement (herein referred to as patient advisors) was considered in priority setting discussions and group

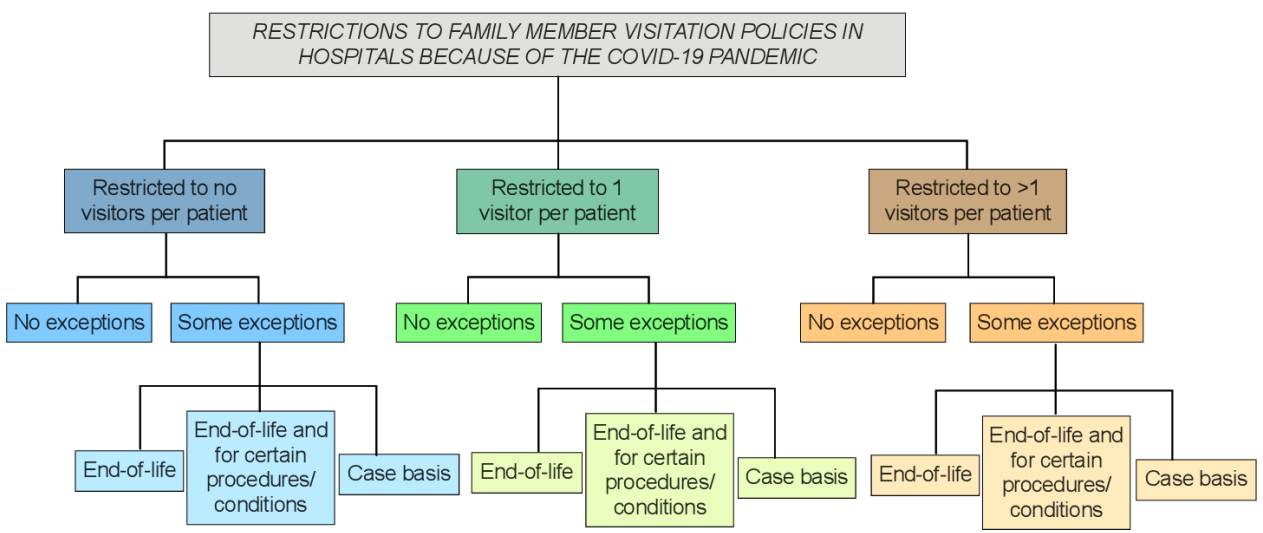

Figure 1 Restricted visitation policy tree. 


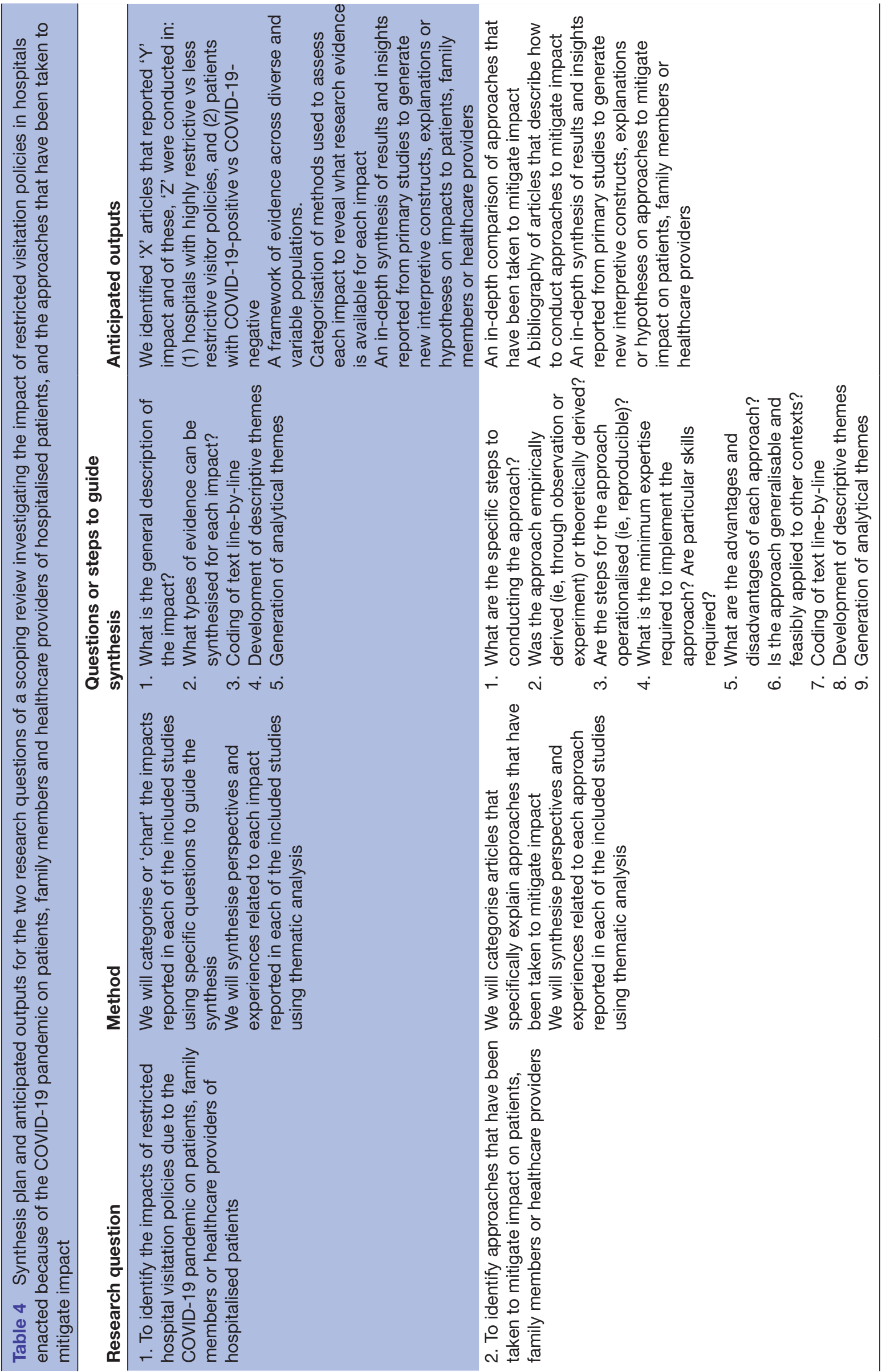


Table 5 Anticipated deliverables from the scoping review

\begin{tabular}{|c|c|c|}
\hline Deliverables & Audience & Methods \\
\hline $\begin{array}{l}\text { Framework of potential impacts } \\
\text { according to degree of visitor } \\
\text { restrictions for hospital to consider prior } \\
\text { to implementing restrictions }\end{array}$ & $\begin{array}{l}\text { Patients, family members, } \\
\text { healthcare providers, researchers } \\
\text { and policy makers }\end{array}$ & $\begin{array}{l}\text { Publish in relevant journals; disseminate at } \\
\text { relevant academic meetings; presentation at } \\
\text { grand rounds }\end{array}$ \\
\hline $\begin{array}{l}\text { A bibliography of articles that describe } \\
\text { how to conduct approaches to mitigate } \\
\text { impact }\end{array}$ & $\begin{array}{l}\text { Patients, family members, } \\
\text { healthcare providers, researchers, } \\
\text { policy makers and funders }\end{array}$ & $\begin{array}{l}\text { Prepare a summary report outlining possible } \\
\text { approaches to mitigate potential impacts that will } \\
\text { include the primary articles, to be made available } \\
\text { as a PowerPoint slide deck; non-traditional } \\
\text { strategies to disseminate our results (eg, social } \\
\text { media, blogs) }\end{array}$ \\
\hline $\begin{array}{l}\text { An in-depth synthesis and comparison } \\
\text { of results and insights on impacts, } \\
\text { including categorisation of methods } \\
\text { used to assess each impact, to reveal } \\
\text { what research is available for each } \\
\text { impact }\end{array}$ & $\begin{array}{l}\text { Patients, family members, } \\
\text { healthcare providers, health } \\
\text { services researchers, policy } \\
\text { makers }\end{array}$ & $\begin{array}{l}\text { Inform a national stakeholder meeting (Delphi } \\
\text { Consensus process) that will generate national } \\
\text { evidence-informed recommendations on } \\
\text { restricted visitation policies informed by } \\
\text { patient, family member and healthcare provider } \\
\text { perspectives and experiences to be implemented } \\
\text { by the Alberta Health Services Critical Care } \\
\text { Strategic Clinical Network, the Canadian Critical } \\
\text { Care Society, the Canadian Society of Respiratory } \\
\text { Therapists, and the Canadian Association of } \\
\text { Critical Care Nurses }\end{array}$ \\
\hline
\end{tabular}

consultations. The patient advisors for this project have worked with our team consenting and recruiting participants for projects in the acute care settings. For the current project, these knowledge users were involved in protocol development and will continue to be involved in the project through to and including dissemination of the published report and implementation of the evidenceinformed recommendations. Our multidisciplinary team of patient advisors and healthcare providers (eg, physician, nurse, respiratory therapist) have established a track record of coleading national peer-reviewed grants and research success in publishing with patient advisors. ${ }^{20}{ }^{21}$ All patient advisors are compensated for their time and are included as coauthors in published work.

\section{Knowledge translation plan}

We will use an integrated knowledge translation approach through the inclusion of knowledge users (ie, patient advisors and multidisciplinary healthcare providers) based on Graham's Knowledge-to-Action framework. ${ }^{22}$ We will disseminate and deliver the products of this review (table 5) to different stakeholder groups using a diverse range of approaches targeted across variable populations and health disciplines. This will allow the opportunity to elicit more generalisable findings that can directly inform practice and policy decisions related to restrictions on family visitation during COVID-19, and for future pandemics.

\section{Anticipated challenges}

The potential benefits of this scoping review must be considered in light of potential challenges. First, we foresee that the yield of the literature searches might be more extensive than anticipated, given the rapid and continuous nature of COVID-19 research in publication or in preprint repositories prior to appearance in journals (eg, medRxiv). We plan to work closely with a health research librarian (ie, information specialist) to ensure that timely completion of the review is feasible. Second, we anticipate challenges related to categorising restricted visitation policies accurately (eg, what are exactly 'some exceptions' might be unclear in the included studies) and to appropriately match potential impacts with the degree of visitor restrictions. Third, we anticipate challenges related to categorising approaches that have been taken to mitigate impact (eg, operationalising steps for each approach). However, we have a strong and diverse team that leads many large studies related to COVID-19 and therefore has the experience and expertise to address these challenges.

\section{DISCUSSION}

The proposed scoping review has the potential to impact practice and policy during the COVID-19 pandemic and will make contributions for future pandemic care responses. Our integrated knowledge translation strategy will ensure rapid and comprehensive synthesis of available evidence to accelerate the development of evidenceinformed recommendations for family visitation during COVID-19 and beyond.

There is presently no comprehensive synthesis of all available research on the impacts of restricted visitation policies during the COVID-19 pandemic. To develop this synthesis, a framework of policies and associated impacts is needed. We aim to develop a framework according to the degree of restriction for hospital visitation policies, as 
well as for patient COVID-19 status. This framework will provide a systematic process for policy makers to make informed decisions about potential impacts of restricted visitation policies in hospitals, and to make informed decisions about which approach is the most appropriate to address and mitigate impact, that will directly inform practice and policy decisions within these groupings.

Our work will help identify gaps in the literature where primary evidence is needed. We anticipate that this scoping review will lead to several subsequent systematic reviews. For example, one future systematic review may focus on diagnosed mental health disorders. We also expect that this review may inform future studies on effective mental health interventions and psychological strategies for patients, family members and healthcare providers impacted by COVID-19, that will enhance the extent and quality of the research evidence base.

Finally, this review will directly inform a national stakeholder meeting that will generate national evidenceinformed recommendations on restricted visitation policies. Our work will provide real-time information for this process by providing a comprehensive summary of patient, family member and healthcare provider perspectives and experiences across diverse populations to establish evidence-informed visitation policies for the COVID-19 pandemic, future pandemics, and for interpandemic periods when family members cannot visit due to frailty or distance.

\section{Author affiliations}

${ }^{1}$ Critical Care Medicine, University of Calgary Cumming School of Medicine, Calgary, Alberta, Canada

${ }^{2}$ Medicine, University of Calgary Cumming School of Medicine, Calgary, Alberta,

Canada

${ }^{3}$ Critical Care Medicine, University of Alberta Faculty of Medicine \& Dentistry, Edmonton, Alberta, Canada

${ }^{4}$ Medicine, University of Toronto Faculty of Medicine, Toronto, Ontario, Canada

${ }^{5}$ Medicine, McMaster University, Hamilton, Ontario, Canada

${ }^{6}$ Nurse Practitioners, Royal Columbian Hospital, New Westminster, British Columbia,

Canada

${ }^{7}$ Sunnybrook Health Sciences Institute, Sunnybrook Research Institute, Toronto,

Ontario, Canada

${ }^{8}$ Psychiatry, University of Manitoba, Winnipeg, Manitoba, Canada

${ }^{9}$ Departments of Pediatrics and Critical Care Medicine, University of British

Columbia, Vancouver, British Columbia, Canada

${ }^{10}$ Medicine, University of Manitoba, Winnipeg, Manitoba, Canada

${ }^{11}$ Psychiatry, University of Calgary Cumming School of Medicine, Calgary, Alberta, Canada

${ }^{12}$ Critical Care Medicine, University of Alberta Faculty of Medicine and Dentistry,

Edmonton, Alberta, Canada

${ }^{13}$ Critical Care Medicine, Chinook Regional Hospital, Lethbridge, Alberta, Canada

${ }^{14}$ Li Ka Shing Knowledge Institute, St. Michael's Hospital, Toronto, Ontario, Canada

${ }^{15}$ Respiratory Therapy, Canadian Society of Respiratory Therapists, Saint John, New Brunswick, Canada

${ }^{16}$ Medicine, Schulich School of Medicine \& Dentistry, Western University, London, Ontario, Canada

\section{Twitter Sofia Ahmed @SofiaAhmedMD and Alison Fox-Robichaud @drfoxrob}

Contributors All those designated as authors have met all four ICMJE criteria for authorship: substantial contributions to the conception or design of the work; or the acquisition, analysis, or interpretation of data for the work; drafting the work or revising it critically for important intellectual content; final approval of the version to be published; agreement to be accountable for all aspects of the work in ensuring that questions related to the accuracy or integrity of any part of the work are appropriately investigated and resolved. HTS, KDK, SA, SMB, TB, KEAB, DJC, SC, CJD, NF, AF-R, RF, MK, SM, DN, KO, KKP, SBP, OR, BR, BGS, AS, KS, SS, SES, AW, JPL and KF applied for and received funding. SJM, HTS, MCA, JPL and KF are the core team leading the systematic review. SJM and KF drafted the initial manuscript for the protocol. HTS, KDK and JPL provided advice at different stages during drafting of the protocol manuscript. All authors approved the initial version of the manuscript that was submitted. SJM and KF revised the manuscript following peer-review. All authors approved the final version of the revised manuscript that was submitted. $\mathrm{KF}$ is the guarantor and attests that all listed authors meet authorship criteria and that no others meeting the criteria have been omitted. SJM and KF affirm that this manuscript is an honest, accurate and transparent account of the study that will be reported; that no important aspects of the study will be omitted; and that any discrepancies from the study as planned (and described in the protocol) will be explained.

Funding This scoping review is funded by the Canadian Institutes of Health Research-Canadian 2019 Novel Coronavirus (COVID-2019) Rapid Research Funding Opportunity-Operating Grant (Grant Number 447521) to KF. The funders had no role in protocol design, data collection and analysis, decision to publish or preparation of the manuscript; no financial relationships with any organisations that might have an interest in the submitted work in the previous three years; no other relationships or activities that could appear to have influenced the submitted work.

Competing interests None declared.

Patient consent for publication Not applicable.

Provenance and peer review Not commissioned; externally peer reviewed.

Supplemental material This content has been supplied by the author(s). It has not been vetted by BMJ Publishing Group Limited (BMJ) and may not have been peer-reviewed. Any opinions or recommendations discussed are solely those of the author(s) and are not endorsed by BMJ. BMJ disclaims all liability and responsibility arising from any reliance placed on the content. Where the content includes any translated material, BMJ does not warrant the accuracy and reliability of the translations (including but not limited to local regulations, clinical guidelines, terminology, drug names and drug dosages), and is not responsible for any error and/or omissions arising from translation and adaptation or otherwise.

Open access This is an open access article distributed in accordance with the Creative Commons Attribution Non Commercial (CC BY-NC 4.0) license, which permits others to distribute, remix, adapt, build upon this work non-commercially, and license their derivative works on different terms, provided the original work is properly cited, appropriate credit is given, any changes made indicated, and the use is non-commercial. See: http://creativecommons.org/licenses/by-nc/4.0/.

ORCID iDs

Stephana J Moss http://orcid.org/0000-0002-1157-884X

Sofia Ahmed http://orcid.org/0000-0003-3000-2229

Christopher J Doig http://orcid.org/0000-0002-8576-9139

Alison Fox-Robichaud http://orcid.org/0000-0001-9912-3606

Oleska Rewa http://orcid.org/0000-0002-8718-0547

Jeanna Parsons Leigh http://orcid.org/0000-0002-8408-674X

Kirsten M Fiest http://orcid.org/0000-0002-7299-6594

\section{REFERENCES}

1 Davidson JE, Aslakson RA, Long AC, et al. Guidelines for FamilyCentered care in the neonatal, pediatric, and adult ICU. Crit Care Med 2017;45:103-28.

2 Family visitation in the adult intensive care unit. Crit Care Nurse 2017;37:88.

3 Nassar Junior AP, Besen BAMP, Robinson CC, et al. Flexible versus restrictive visiting policies in ICUs: a systematic review and metaanalysis. Crit Care Med 2018;46:1175-80.

4 Westphal GA, Moerschberger MS, Vollmann Dailany D'Aroz, et al. Effect of a 24-h extended visiting policy on delirium in critically ill patients. Intensive Care Med 2018;44:968-70.

5 Subramanian S, Pamplin JC, Hravnak M, et al. Tele-Critical care: an update from the Society of critical care medicine Tele-ICU Committee. Crit Care Med 2020;48:553-61.

6 Dhala A, Sasangohar F, Kash B, et al. Rapid implementation and innovative applications of a virtual intensive care unit during the COVID-19 pandemic: case study. J Med Internet Res 2020;22:e20143. 
7 Burns KEA, Devlin JW, Hill NS. Patient and family engagement in designing and implementing a weaning trial: a novel research paradigm in critical care. Chest 2017;152:707-11.

8 Sasangohar F, Jones SL, Masud FN, et al. Provider burnout and fatigue during the COVID-19 pandemic: lessons learned from a highvolume intensive care unit. Anesth Analg 2020;131:106-11.

9 Galea S, Merchant RM, Lurie N. The mental health consequences of COVID-19 and physical distancing: the need for prevention and early intervention. JAMA Intern Med 2020;180:817-8.

10 Duan L, Zhu G. Psychological interventions for people affected by the COVID-19 epidemic. Lancet Psychiatry 2020;7:300-2.

11 Xiang Y-T, Yang Y, Li W, et al. Timely mental health care for the 2019 novel coronavirus outbreak is urgently needed. Lancet Psychiatry 2020;7:228-9.

12 Arksey H. Scoping the field: services for carers of people with mental health problems. Health Soc Care Community 2003;11:335-44.

13 Joanna Briggs Institute. The Joanna Briggs Institute best practice information sheet: the effectiveness of pelvic floor muscle exercises on urinary incontinence in women following childbirth. Nurs Health Sci 2011:13:378-81.

14 Moher D, Shamseer L, Clarke M, et al. Preferred reporting items for systematic review and meta-analysis protocols (PRISMA-P) 2015 statement. Syst Rev 2015;4:1.
15 Tricco AC, Lillie E, Zarin W, et al. PRISMA extension for scoping reviews (PRISMA-ScR): checklist and explanation. Ann Intern Med 2018;169:467-73.

16 McGowan J, Sampson M, Salzwedel DM, et al. PRESS peer review of electronic search strategies: 2015 Guideline Statement. J Clin Epidemiol 2016;75:40-6.

17 Thomas J, Harden A. Methods for the thematic synthesis of qualitative research in systematic reviews. BMC Med Res Methodol 2008;8:45.

18 Farrier CE, Stelfox HT, Fiest KM. In the pursuit of partnership: patient and family engagement in critical care medicine. Curr Opin Crit Care 2019;25:505-10.

19 Canadian Institutes of Health Research. Strategy for patient-oriented Research capacity development framework [Online], 2015. Available: http://www.cihr-irsc.gc.ca/e/49307.html [Accessed Jan 2021].

20 Gill M, Bagshaw SM, McKenzie E, et al. Patient and family memberled research in the intensive care unit: a novel approach to patientcentered research. PLoS One 2016;11:e0160947.

21 Krewulak KD, Sept BG, Stelfox HT, et al. Feasibility and acceptability of family administration of delirium detection tools in the intensive care unit: a patient-oriented pilot study. CMAJ Open 2019;7:E294-9.

22 Graham ID, Logan J, Harrison MB, et al. Lost in knowledge translation: time for a MAP? J Contin Educ Health Prof 2006;26:13-24. 